\title{
Weaning from veno-venous extracorporeal membrane oxygenation: how I do it
}

\author{
Lars M. Broman ${ }^{1,2}$, Maximilian V. Malfertheiner ${ }^{1,3}$, Andrea Montisci ${ }^{4}$, Federico Pappalardo ${ }^{1,5}$ \\ ${ }^{1}$ European ECMO Advisory Board; ${ }^{2}$ ECMO Centre Karolinska, Karolinska University Hospital, and Department of Physiology and Pharmacology, \\ Karolinska Institutet, Stockholm, Sweden; ${ }^{3}$ Department of Internal Medicine II, Cardiology and Pneumology, University Medical Center \\ Regensburg, Regensburg, Germany; ${ }^{4}$ Cardiothoracic Centre, Instituto Clinico Sant'Ambrogio, Gruppo Ospedaliero San Donato, Milan, Italy; \\ ${ }^{5}$ Department of Anesthesia and Intensive Care, San Raffaele Scientific Institute, Vita-Salute University, Milan, Italy \\ Contributions: (I) Conception and design: None; (II) Administrative support: None; (III) Provision of study materials or patients: None; (IV) \\ Collection and assembly of data: LM Broman, F Pappalardo, MV Malfertheiner; (V) Data analysis and interpretation: All authors; (VI) Manuscript \\ writing: All authors; (VII) Final approval of manuscript: All authors. \\ Correspondence to: Federico Pappalardo. Department of Anesthesia and Intensive Care, San Raffaele Scientific Institute, Milan, Italy. \\ Email: Pappalardo.federico@hsr.it.
}

\begin{abstract}
Veno-venous extracorporeal membrane oxygenation (VV ECMO) is a rescue treatment for acute respiratory distress syndrome (ARDS) failing protective mechanical ventilation. It temporarily provides proper gas exchange: hypoxia is treated by adjusting the blood flow rate and fraction in spired oxygen over the ventilator $\left(\mathrm{FiO}_{2}\right)$ on the extracorporeal membrane oxygenation (ECMO) circuit while $\mathrm{CO}_{2}$ removal is regulated by the ECMO fresh gas flow. Therefore, ventilator settings can be gradually reduced allowing the lungs to rest and recover. Nowadays, indications for ECMO referral and implantation are clearly formulated; on the contrary, little evidence currently exists to guide the process of weaning from ECMO support, especially concerning the timing during the course of lung healing. Therefore, indications to stop ECMO are less well standardized so that in clinical trials extracorporeal assistance is generally continued until lung recovery, with neither specific nor homogenous criteria for withdrawal. Notably, in almost all papers dealing with data on VV ECMO support, the management of weaning and the weaning procedure itself are not described. The aim of this paper is to make a picture of VV ECMO weaning, as it is performed in three European large volume intensive care units (ICUs) which represent referral centers for VV ECMO treatment. We focused on data concerning the timing of VV ECMO weaning and parameters at the time of weaning, in order to assess adequacy and safety of VV ECMO removal.
\end{abstract}

Keywords: Weaning; veno-venous (VV); extracorporeal membrane oxygenation (ECMO)

Submitted Sep 04, 2017. Accepted for publication Sep 06, 2017.

doi: $10.21037 /$ jtd.2017.09.95

View this article at: http://dx.doi.org/10.21037/jtd.2017.09.95

\section{Introduction}

Veno-venous extracorporeal membrane oxygenation (VV ECMO) is a rescue therapy for the most severe cases of respiratory failure. Criteria to start a patient on VV ECMO are published by ELSO (Extracorporeal Life Support Organization, Ann Arbor, MI, USA. www.elso.org) (1) and have been adopted with only slight variations by most ECMO centers. Once a patient is on VV ECMO for respiratory failure the purpose of the device is to sustain gas exchange and allow the injured lung to recover by reducing the need for applied pressures of mechanical ventilation. Ideally the process to wean a patient off from VV ECMO therapy starts once the lungs start to improve, which can be seen by improving compliance and better gas exchange (Figure 1).

The weaning process from the two modalities of ECMO, veno-arterial (VA) ECMO and VV ECMO, respectively, 


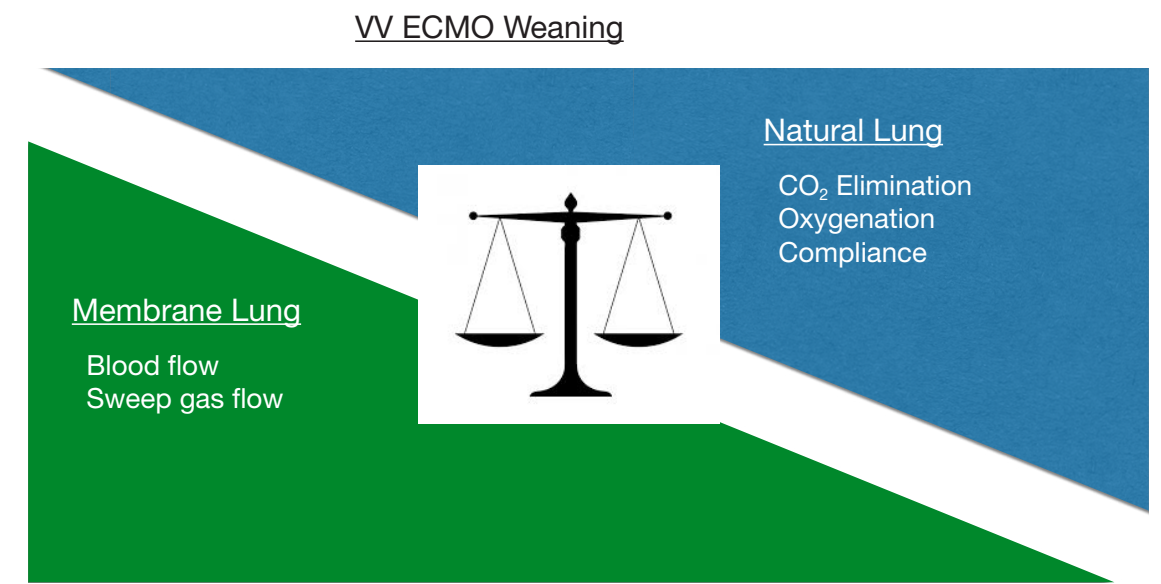

Figure 1 Veno-venous ECMO weaning starts with improvement of the natural lung. Improving gas exchange should be monitored and the weaning process should focus on keeping the balance between natural and membrane lung. ECMO, extracorporeal membrane oxygenation.

are rather different (2-4). In the former both cardiac and pulmonary recovery have to be assessed before decision to decannulate. For VV ECMO the procedure is more straight-forward. Nevertheless, there are great differences in how the weaning process is managed from center to center. These differences may also be illustrated by published guidelines available on the internet (ELSO/www. elso.org) (1), with a bias towards adults and, of course, the more complicated VA ECMO.

The aim of the current overview is to describe experiences from different approaches to how weaning off from VV ECMO could be undertaken in safe and at the same time pragmatic ways.

Three experienced ECMO centers describe their approach to VV ECMO weaning in acute lung failure.

\section{Settings}

ECMO Centre Karolinska, Stockholm, became the second ECMO unit in Europe established in 1987 at a pediatric intensive care unit (PICU), and has been an ELSO Center of Excellence since 2007. The unit is dedicated to ECMO treatments, and all ages and diagnoses except postcardiotomy are treated. ECMO is performed by ECMO specialists (PICU/ICU nurse) and ECMO physicians (anesthetists and intensivists). Both categories have passed an ELSO accredited course. Catheterization are performed by a cannulating surgeon. The basal staffing is one ECMO-specialist (ICU nurse), 1/2 ECMO Physician and $1 / 2$ Nurse's aid per patient. The patient is never left alone. Approximately 90 annual ECMO runs are performed, and $55 \%$ are commenced on VA ECMO. A total of 1,200 patients have been treated up today. Integrated in the clinic is also an organization for mobile ECMO that have performed close to 900 national and international primary and secondary transports on ECMO.

After commencement of ECMO the ventilator pressures and fraction in spired oxygen over the ventilator $\left(\mathrm{FiO}_{2}\right)$ are reduced to "lung rest settings", aiming to keep the lung open. This is also a matter of patient safety since the majority $(80 \%)$ of the patients have a transfer ahead. After arrival to the ECMO ICU, $\mathrm{FiO}_{2}$ may be in the range of $35-50 \%$, and most patients supported with pressure control or pressure support mode depending on course of illness and patient comfort. In children, positive end expiratory pressure (PEEP) usually is set to $3-5 \mathrm{cmH}_{2} \mathrm{O}$, and the driving pressure to $10-20 \mathrm{cmH}_{2} \mathrm{O}$. For adults, the corresponding pressures would be $6-12 \mathrm{cmH}_{2} \mathrm{O}$ and $15-25 \mathrm{cmH}_{2} \mathrm{O}$, respectively. Blood gases are taken every hour and the aim for oxygenation is oxygen saturation $\left(\mathrm{SaO}_{2}\right)$ $>85 \%$, and partial pressure for dissolved carbon dioxide in plasma $\left(\mathrm{pCO}_{2}\right)$ corresponding to a normal $\mathrm{pH}$. Tracheostomy is performed in the adult at day two or three after which sedation is reduced to awake the patient. Pediatric and neonatal patients are not tracheostomized but sedation is reduced. Fluid balance is often an issue and most of the patients are submitted to continuous renal replacement therapy (CRRT) with fluid balance as the main indication.

The University Hospital Regensburg provides ECMO service since about 20 years and was the first German ECMO centre to receive the ELSO award of Excellence in Life support in 2014. Within the University Hospital 
Regensburg ECMO is performed on different specialized ICU's: medical, cardiac surgery, and general surgical. Most cases of severe respiratory failure receiving VV ECMO are treated on the medical ICU, however. Approximately 140 annual ECMO runs are performed at the ECMO Center Regensburg, and $60 \%$ are commenced on VV ECMO. Staffing on the ICU for ECMO patients is $1 / 2$ ICU nurse with experience in ECMO patient care, approximately $30 \mathrm{~min} /$ day of a physiotherapist and a minimum of one physician on the ward (max. 15 beds). After implementation of the ECMO, invasiveness of ventilation is reduced to diminish further ventilator-induced lung injury, however PEEP is not reduced to avoid atelectasis due to small TV. The aim for oxygenation is a partial pressure of oxygen $\left(\mathrm{PaO}_{2}\right)$ of $>8 \mathrm{kPa}[>60 \mathrm{mmHg}]$ and partial pressure of carbon dioxide $\left(\mathrm{PaCO}_{2}\right)$ is adjusted to achieve a normal $\mathrm{pH}$ level. Arterial blood gases are routinely taken every 4 hours, and blood gases from both sides of the membrane lung (ML) are obtained at least once daily.

San Raffaele Hospital has started its ECMO activity on 2009 and the workload has been steadily growing up to 125 cases in 2016; $15 \%$ of them are in the VV mode. All patients are treated in a Cardiothoracic ICU which is staffed 24/7 with intensivists competent in extracorporeal supports. All cannulations are performed at bedsides by intensivists with transesophageal echocardiography (TEE) guidance. Dual lumen cannulation is the routine strategy since 2012 . Staffing on the ICU for ECMO patients is $1 / 2$ ICU nurse with experience in ECMO patient care, approximately $30 \mathrm{~min} /$ day of a physiotherapist and a minimum of one physician on the ward. A perfusionist is available 24/7; pre and post ML blood gases are taken at least once daily.

\section{Results}

\section{MO Centre Karolinska}

The weaning process from VV ECMO starts as soon as the tidal volumes start to recover. The ECMO blood flow has usually been reduced over the preceding days as well. The marker for progression is more to carbon dioxide clearance than oxygenation. The process is based on respiratory physiology. For sweep gas, oxygen $\left(\mathrm{FiO}_{2} 1.0\right)$, or oxygen with a mix of carbon dioxide $(0-5 \%)$ is used. The ECMO specialist adjusts the sweep gas flow accordingly to blood gas assessments and daily pre-set goals for $\mathrm{pH}$ and $\mathrm{pCO}_{2}$. During the ECMO run hourly blood gases are sampled from the patient's arterial line and/or the pre ML limb of the ECMO circuit. As the lung recovers and the clearance of $\mathrm{CO}_{2}$ by the native lung increases, the sweep gas flow will successively be reduced. When a ML size-related minimal sweep gas flow is accomplished (adult: $2 \mathrm{~L} / \mathrm{min}$; pediatric/ neonatal: $1 \mathrm{~L} / \mathrm{min}$ ), incremental $\mathrm{pCO}_{2}$ will be added to the sweep gas. The maximum fraction delivered $\mathrm{CO}_{2}\left(\mathrm{FDCO}_{2}\right)$ to the $\mathrm{ML}$ would correspond to $5 \mathrm{kPa}$ [37.5 torr] ambient pressure.

The efficiency of the native lung to clear $\mathrm{CO}_{2}$ is assessed by simultaneous samplings of blood gases before and after the ML. When the $\mathrm{pCO}_{2}$ difference (pre- and post$\mathrm{ML})$ is less than $0.2-0.4 \mathrm{kPa}$ (1.5-3.0 torr), the patient is regarded as "equilibrated". It means that the ML neither clears or adds $\mathrm{CO}_{2}$ to the patient's blood. All $\mathrm{CO}_{2}$ produced by the patient is thus cleared by the patient's native lung. If the arterial $\mathrm{SaO}_{2}$ is adequate, a trial off is performed: $\mathrm{FiO}_{2}$ is set to $35-55 \%$, and the sweep gas discontinued. If the procedure turns out well the sweep may be turned off for hours or overnight if necessary (till decannulation). Otherwise, the sweep gases are turned on again a new assessment will follow the next day.

\section{University Hospital Regensburg}

The weaning process starts after successful treatment of the underlying disease and improvement of lung function $\left[\mathrm{FiO}_{2}\right.$ $<0.45$, PEEP $<10 \mathrm{cmH}_{2} \mathrm{O}$, peak inspiratory pressure (PIP) $<27 \mathrm{cmH}_{2} \mathrm{O}$ ]. When the ventilator settings still allow a protective ventilation and $\mathrm{CO}_{2}$ clearance through the native lung starts to increase the ECMO gas flow can be tapered to adjust $\mathrm{pH}$ values. Whenever ECMO settings are adjusted a blood gas control will be performed within 30 to 60 mins of adjustment. The extracorporeal blood flow is stepwise reduced to $1.5 \mathrm{~L} / \mathrm{min}$. Gas flow is tapered mostly in parallel to the blood flow and finally shut off for 30-60 minutes. If blood gases remain stable and the patient does not develop dyspnoea or tachypnoea the decision to remove the system is made. Depending on the current state of anticoagulation of the patient heparin treatment is stopped and activated partial thromboplastin time (aPTT), platelet count and INR are tested before the ECMO system is removed and decannulation with manual compression is carried out.

\section{San Raffaele Hospital}

After VV ECMO is implanted, the patient is kept sedated over the next $24 \mathrm{~h}$ in order to re-perfuse and re-establish metabolic homeostasis; respiratory rate is decreased to 
$10 / \mathrm{min}$ and TV kept at $6 \mathrm{~mL} / \mathrm{kg}, \mathrm{FiO}_{2}$ is set at the lowest value in order to obtain oxygen saturation by pulse oximetry $\left(\mathrm{SpO}_{2}\right)>90 \%$. Thereafter, if the patient is stable without cardiovascular impairment, sedation is stopped and the ventilator switched off, with the patient connected to a high flow continuous positive airway pressure (CPAP) with pressure set at similar values as PEEP on the ventilator. The patient can be apnoeic for a certain period of time. Once awake, dyspnoea is assessed and, if absent, we wait for full neurological recovery and eventually proceed to extubation. VV ECMO is kept at full support. Once there is clinical improvement on chest $\mathrm{X}$-ray , ECMO flow is progressively reduced to $2.5-3 \mathrm{~L} / \mathrm{min}$ and then sweep gas and $\mathrm{FiO}_{2}$ tapered. After a successful 'trial off', anticoagulation is stopped and cannulas removed with manual compression.

In cases with septic shock and/or multiple organs dysfunction failure (MOF), the process of weaning is strongly dictated by the pattern of recovery of end organs, namely central and peripheral nervous system, and care is decided accordingly. Early tracheostomy is strongly advocated is this scenario.

If the patient succeeds to extubation, non-invasive ventilation with face mask is systematically delivered. On top of that, oral feeding and mobilization can be easily achieved.

\section{Discussion}

Once ECMO has been established in severe acute respiratory distress syndrome (ARDS) patients, a still open question is when and how both extracorporeal and ventilation support could be discontinued safely. It has to acknowledge that several patients die after and despite being weaned. Thus, we could argue that, if not standardized, the withdrawal criteria applied might be inappropriate and imprudent. Moreover, the re-institution of ECMO and/or mechanical ventilation, albeit infrequent, is usually associated with poor outcome. This emphasizes the relevance of proper understanding how both lung disease and general clinical conditions are evolving and, consequently, what is the potential role of ECMO for each specific patient as bridge to recovery, to transplant, or to "nowhere", in the attempt to establish appropriateness and timing for both extracorporeal and ventilatory support discontinuation (5-6).

Differences in weaning strategies for VV ECMO are based on expert opinions rather than evidence. This fact may show that weaning can be done in many ways. While some centres keep the blood flow of the ECMO device high $(>3 \mathrm{~L} / \mathrm{min})$ to prevent any additional clotting within the systems there are also centres that reduce the blood flow to a minimum of $1 \mathrm{~L} / \mathrm{min}$ (adults), following the idea of reduced shear stress for the circulated blood. The blood flow per se is of no concern in VV ECMO after the sweep gas has been turned off since it is isovolumic.

Using a physiologic Karolinska approach to wean is a pragmatic way of indirectly start to "wean" as soon as the tidal volume start to recover. The $\mathrm{CO}_{2}$ clearance capacity of the patient's lung is continuously assessed during the recovery phase. The risk with the $\mathrm{CO}_{2}$ approach would be carbon dioxide narcosis if the partial pressure of $\mathrm{CO}_{2}$ in the sweep gas increase to a load above the limit of total $\mathrm{CO}_{2}$ clearance (sum of native and ML capacity). To our knowledge this has never occurred.

In the literature including published guidelines, different strategies have been described $(1-4,7)$ where either a trial off is performed or not. The weaning process itself does not seem to be controversial.

It remains to be understood whether the preferable approach is whether to stop ECMO or not when the patient is still mechanically ventilated. Ventilatory strategies attempting to enable spontaneous breathing and, thus, encouraging earlier weaning from mechanical ventilation could prevent the complications of sedation as well as muscle deconditioning and fatigue, which may contribute to prolong the duration of ventilatory support itself. Indeed, early extubation is an alternative briefly suggested by the ELSO guidelines and it may turn beneficial allowing to avoid the risk of ventilation-induced lung injury and ventilator-associated pneumonia, both potentially leading to exacerbation of the initial lung tissue damage. Recently, this topic has been gaining interest (8), but the evidence available so far is still limited or specific to subsets of patients, particularly end-stage respiratory disease awaiting lung transplantation (9-12). This makes it insufficient to definitively assess whether potential benefits could balance the risks arising from perhaps longer duration of ECMO support. The idea to reduce ECMO flow to $2.5-3 \mathrm{~L} / \mathrm{min}$ stems from the assumption that at this configuration the mechanical performance of the circuit would be the best without risks for stagnation and clotting, nor blood trauma.

On top of that, several adjuvant strategies have been proposed to "speed up" time of recovery, i.e., time to wean or increase the margins to come off ECMO. Levosimendan may increase the power and strength of the diaphragm $(13,14)$, and does also seem to improve recovery to wean 
after lung transplantation (15). In sheep submitted to ECMO, triiodothyronine (T3) decrease significantly: supplementation of T3 seems to restore lactate metabolism, improve metabolic flux, and enhance diaphragm contractile function during weaning $(9,16)$. The conversion to T3 from T4 is dependent on the seleno-enzyme deiodinase-2 (DI2). Instead of hormonal treatment, micronutrients may suffice: in critical illness, it has been shown that serum selenium decrease with a concomitant decrease of glutathione peroxidase activity (GPX). High-dose substitution of selenium restores GPX activity in both the newborn, child and adult (17-19). The physiology may be similar for the DI2 activity. Kimmoun et al. (20) described that proning may shorten time for weaning. However, echocardiography does not seem to push weaning in VV ECMO (21).

In the light of these findings, further investigations are necessary in order to standardize the weaning process from VV ECMO. Now that VV ECMO treatment is consolidated and indications for implantation have been clarified (22), the efforts should be focused on the identification of the most appropriate strategy for weaning, and its timing.

\section{Acknowledgements}

None.

\section{Footnote}

Conflicts of Interest: LM Broman, MV Malfertheiner and $\mathrm{F}$ Pappalardo are members of the independent research consortium European ECMO Advisory Board, the EuroELSO Workgroup for Innovative Technologies, and the Medical Advisory Board of Eurosets Medical Srl, Medolla, Italy. The other author has no conflicts of interest to declare.

\section{References}

1. ELSO Guidelines. Extracorporeal Life Support Organization, Ann Arbor, MI, USA. Available online: http://www.elso.org

2. Short BL, Williams L. editors. ECMO Specialist training manual 3rd ed. Extracorporeal Life Support Organization, Ann Arbor, MI, USA.

3. Annich GM, Lynch WR, Maclaren G, et al. editors. Red book 4th ed. Extracorporeal Life Support Organization, Ann Arbor, MI, USA.
4. Brogan TV, Lequier L, Lorusso R, et al. editors. Red book 5th ed. Extracorporeal Life Support Organization, Ann Arbor, MI, USA.

5. Ma DS, Kim JB, Jung SH, et al. Outcomes of venovenous extracorporeal membrane oxygenation support for acute respiratory distress syndrome in adults. Korean J Thorac Cardiovasc Surg 2012;45:91-4.

6. Sajjad M, Osman A, Mohsen S, et al. Extracorporeal membrane oxygenation in adults: experience from the Middle East. Asian Cardiovasc Thorac Ann 2013;21:521-7.

7. The Alfred Health Guideline on Extracorporeal Membrane Oxygenation (ECMO). Available online: http:// www.alfredicu.org.au/assets/Documents/ICU-Guidelines/ ECMO/ECMOGuideline.pdf

8. Schmidt M, Pellegrino V, Combes A, et al. Extracorporeal membrane oxygenation in awake patients as bridge to lung transplantation. Am J Respir Crit Care Med 2012;185:763-8.

9. Olsson KM, Simon A, Strueber M, et al. Extracorporeal membrane oxygenation in nonintubated patients as bridge to lung transplantation. Am J Transplant 2010;10:2173-8.

10. Schmidt F, Sasse M, Boehne M, et al. Concept of "awake venovenous extracorporeal membrane oxygenation" in pediatric patients awaiting lung transplantation. Pediatr Transplant 2013;17:224-30.

11. Nosotti M, Rosso L, Tosi D, et al. Extracorporeal membrane oxygenation with spontaneous breathing as a bridge to lung transplantation. Interact Cardiovasc Thorac Surg 2013;16:55-9.

12. Doorduin J, Sinderby CA, Beck J, et al. The calcium sensitizer levosimendan improves human diaphragm function. Am J Respir Crit Care Med 2012;185:90-5.

13. Van Hees HW, Dekhuijzen PN, Heunks LM. Levosimendan enhances force generation of diaphragm muscle from patients with chronic obstructive pulmonary disease. Am J Respir Crit Care Med 2009;179:41-7.

14. Feltracco P, Carollo C, Ori C. Levosimendan in lung transplant recipients with difficult weaning from ECMO. Minerva Anestesiol 2015;81:92-3.

15. Kajimoto M, Ledee DR, Xu C, et al. Triiodothyronine activates lactate oxidation without impairing fatty acid oxidation and improves weaning from extracorporeal membrane oxygenation. Circ J 2014;78:2867-75.

16. Files MD, Kajimoto M, O'Kelly Priddy CM, et al. Triiodothyronine facilitates weaning from extracorporeal membrane oxygenation by improved mitochondrial substrate utilization. J Am Heart Assoc 2014;3:e000680. 
17. Angstwurm MW, Engelsmann L, Zimmermann T, et al. Selenium in intensive care (SIC) study: results of a prospective randomized, placebo-controlled, multiplecenter study in patients with severe systemic inflammatory response syndrome, sepsis and septic shock. Crit Care med 2007;35:118-26.

18. Woth G, Nagy B, Mérei Á, et al. The effect of Na-selenite treatment on the oxidative stress-antioxidants balance of multiple organ failure. J Crit Care 2014;29:883.e7-11.

19. Broman LM, Lindfors M, Hebert C, et al. Low concentration of serum selenium is associated with the severity of multiple organ failure in critically ill children. Clin Nutr 2017 21. pii: S0261-5614 30225-X.

Cite this article as: Broman LM, Malfertheiner MV, Montisci A, Pappalardo F. Weaning from veno-venous extracorporeal membrane oxygenation: how I do it. J Thorac Dis 2018;10(Suppl 5):S692-S697. doi: 10.21037/jtd.2017.09.95
20. Kimmoun A, Guerci P, Bridey C, et al. Prone positioning use to hasten veno-venous ECMO weaning in ARDS. Intensive Care Med 2013;39:1877-9.

21. Guinot PG, Soulignac C, Zogheib E, et al. Interactions between veno-venous extracorporeal membrane oxygenation and cardiac function: an echocardiographic study upon weaning. Br J Anaesth 2016;117:821-2.

22. Malfertheiner MV, Broman LM, Belliato M, et al. Management strategies in venovenous extracorporeal membrane oxygenation: a retrospective comparison from five European centres. Crit Care Resusc 2017;19(Suppl 1):76-81. 